\title{
DESIGN AND IMPLEMENTATION OF ELECTRONICS AND DATA ACQUISITION SYSTEM FOR ULTRA-FAST FLASH OBSERVATORY
}
A. Jung ${ }^{1,2}$, S. Ahmad ${ }^{3}$, P. Barrillon ${ }^{3}$, S. Brandt ${ }^{4}$, C. Budtz-Jørgensen ${ }^{4}$, A.J. Castro-Tirado ${ }^{5}$, S.-H. Chang ${ }^{6}$, Y.-Y. Chang ${ }^{7}$, C.R. Chen ${ }^{6}$,
P. Chen ${ }^{7}$, H.S. Choi $^{8}$, Y.J. Choi ${ }^{9}$, P. Connell ${ }^{10}$, S. Dagoret-Campagne ${ }^{3}$, C. Eyles ${ }^{10}$, B. Grossan ${ }^{11}$, J.J. Huang ${ }^{7}$, M.-H.A. Huang ${ }^{12}$, S. Jeong ${ }^{1,2}$, J.E. Kim ${ }^{1,2}$, M. Kim ${ }^{13}$, S.-W. Kim ${ }^{14}$, Y.W. Kim ${ }^{13}$, A.S. Krasnov ${ }^{15}$, J. Lee ${ }^{13}$, H. $\operatorname{Lim}^{13}$, C.-Y. Lin ${ }^{6}$, E.V. Linder ${ }^{2,11,16}$, T.-C. Liu ${ }^{7}$, N. Lund ${ }^{4}$, J.W. Nam ${ }^{7}$, K.W. Min ${ }^{9}$, G.W. Na ${ }^{1,2}$, M.I. Panasyuk ${ }^{15}$, I.H. Park ${ }^{13}$, V. Reglero ${ }^{10}$, J. Ripa ${ }^{13}$, J.M. Rodrigo ${ }^{10}$, G.F. Smoot ${ }^{2,11,16}$, J.E. Suh ${ }^{2}$, S. Svertilov ${ }^{15}$, N. Vedenkin ${ }^{15}$, M.-Z. Wang ${ }^{7}$ and I. Yashin ${ }^{15}$, on behalf of the UFFO collaboration

\begin{abstract}
The Ultra-Fast Flash Observatory (UFFO) Pathfinder for Gamma-Ray Bursts (GRBs) consists of two telescopes. The UFFO Burst Alert \& Trigger Telescope (UBAT) handles the detection and
\end{abstract}

\footnotetext{
${ }^{1}$ Institute of Basic Science, Sungkyunkwan University, Suwon, South Korea; e-mail: erajung@gmail.com

2 Department of Physics, Ewha Womans University, Seoul, South Korea

${ }^{3}$ LAL, University of Paris-Sud 11, Orsay, France

4 Technical University of Denmark, Copenhagen, Denmark

5 Instituto de Astrofísica de Andalucía, CSIC, Granada, Spain

6 National Space Organization, Taiwan

7 LeCosPA, National Taiwan University, Taipei, Taiwan

8 Korea Institute of Industrial Technology, Cheonan, South Korea

9 Department of Physics, Korea Advanced Institute of Science and Technology, Daejeon, South Korea

10 University of Valencia, Valencia, Spain

11 University of California at Berkeley, Space Sciences Laboratory, USA

12 National United University, Miao-Li, Taiwan

13 Sungkyunkwan University, Suwon, South Korea

14 Department of Astronomy, Yonsei University, Seoul, South Korea

15 SINP, Moscow State University, Moscow, Russia

16 Institute for Early Universe, Ewha Womans University, Seoul, South Korea
} 
localization of GRBs, and the Slewing Mirror Telescope (SMT) conducts the measurement of the UV/optical afterglow. UBAT is equipped with an X-ray detector, analog and digital signal readout electronics that detects X-rays from GRBs and determines the location. SMT is equipped with a stepping motor and the associated electronics to rotate the slewing mirror targeting the GRBs identified by UBAT. First the slewing mirror points to a GRB, then SMT obtains the optical image of the GRB using the intensified CCD and its readout electronics. The UFFO Data Acquisition system (UDAQ) is responsible for the overall function and operation of the observatory and the communication with the satellite main processor. In this paper we present the design and implementation of the electronics of UBAT and SMT as well as the architecture and implementation of UDAQ.

\section{Introduction}

Gamma-Ray Bursts (GRBs) are flashes of gamma ray flux originating from the most energetic events in the sky, with peak photon luminosities higher than that of any other object in the Universe (Jung et al. 2011). As such the early-stage observations of the light curves and emission spectrum are essential to the understanding of the nature of GRBs. However, the early-phase observations were often limited by the response time of the instruments operated in space. For example, in the case of the Swift observatory the entire spacecraft has to be maneuvered to point toward the GRB position after being triggered by the detection of $\gamma$-rays, which typically takes about a minute. Thus, it is difficult to obtain sub-minute ultraviolet (UV) and visible light curves with Swift (Park et al. 2013). The proposed Ultra-Fast Flash Observatory Pathfinder (UFFO-P) is designed to observe sub-minute GRB light curves by adopting the slewing mirror technology (Jeong et al. 2013). This removes the need to repoint the entire spacecraft.

\section{Overview of Ultra-Fast Flash Observatory Pathfinder (UFFO-P)}

One of the key aspects of the upcoming UFFO-P for GRBs identification is the electronics, which is based on a novel space telescope technique. The main goal of UFFO-P is to systematically measure early UV/optical photons from GRBs up to sub-second timescales, in order to gain a deeper understanding of GRB mechanisms. UFFO-P is planned to be launched by on the Lomonosov spacecraft in 2013 into a sun-synchronous orbit at an altitude of $\sim 550 \mathrm{~km}$. As shown in Figure 1, UFFO-P consists of two telescopes and one main data acquisition system: the UFFO Burst Alert \& Trigger Telescope (UBAT) for the detection and location of GRBs, the Slewing Mirror Telescope (SMT) aimed at measuring the UV/optical afterglow, and the UFFO Data Acquisition (UDAQ) system for controlling the operations and communications with the telescopes.

\subsection{UFFO Burst Alert \& Trigger Telescope (UBAT)}

UBAT is designed to detect the hard X-ray photons from GRBs in the energy range from $\sim 15$ to $\sim 100 \mathrm{keV}$. UBAT is comprised of a mechanical assembly, a coded mask 


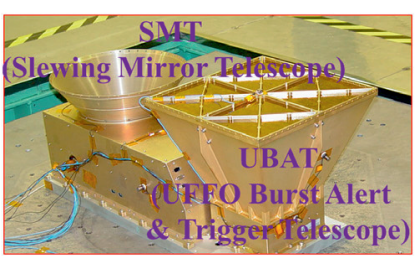

UFFO Pathfinder

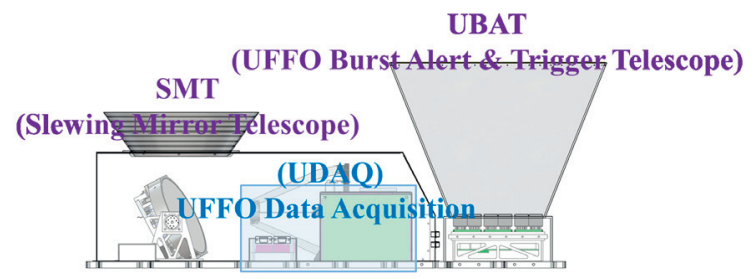

Fig. 1. Left: manufactured UFFO-P. Right: drawing.

with a hopper and a detector. The detector includes the YSO crystals, MAPMTs, FPCB, analog and digital boards, high voltage boards, and the support structure, as can be seen in Figure 2 left.
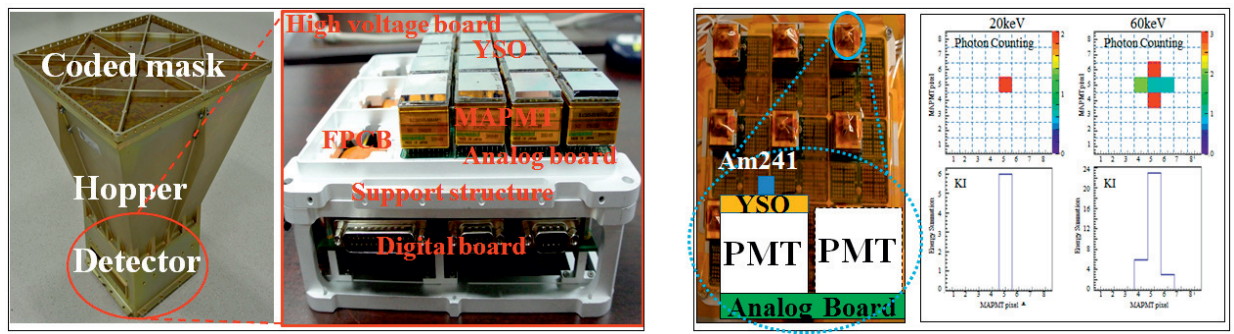

Fig. 2. Left: UBAT and detector. Right: test set up and result of test [4].

The YSO scintillation crystal converts the $\gamma /$ X-ray photons to UV photons, which eventually become electrical pulses through the chain of multi-anode photomultipliers (MAPMTs) and pulse-shaping electronics. These electrical pulses are measured and recorded with a period of $5 \mu \mathrm{s}$ (Jung et al. 2011). The 64-channel MAPMT is read out by SPACIROC (Spatial Photomultiplier Array Counting and Integrating Chip) ASIC (Ahmad et al. 2010). The data are transferred to a digital board built around an Actel A3PE3000L Field-Programmable Gate Array (FPGA) which provides the computing power for trigger decisions. The main components of UFFO-P are shown in Table 1. As seen in Figure 2 right, Americium-241 (Am241) is placed on the YSO crystal of one pixel. The radiation from the Am241 $20 \mathrm{keV}$ and $60 \mathrm{keV}$ lines are observed.

\subsection{Slewing Mirror Telescope (SMT)}

As shown in Figure 3, SMT is an ultra-fast optical/UV telescope that can slew to a target within 1 sec. The detector is an intensified CCD with Micro-Channel Plates (MCPs). SMT has a readout rate of $4 \mathrm{~ms}$ and can acquire 250 frames per second. It takes only 1s to receive the trigger signal to slew the motorized mirror towards the target and collect UV/optical data (Kim et al. 2011). The CCD readout, 


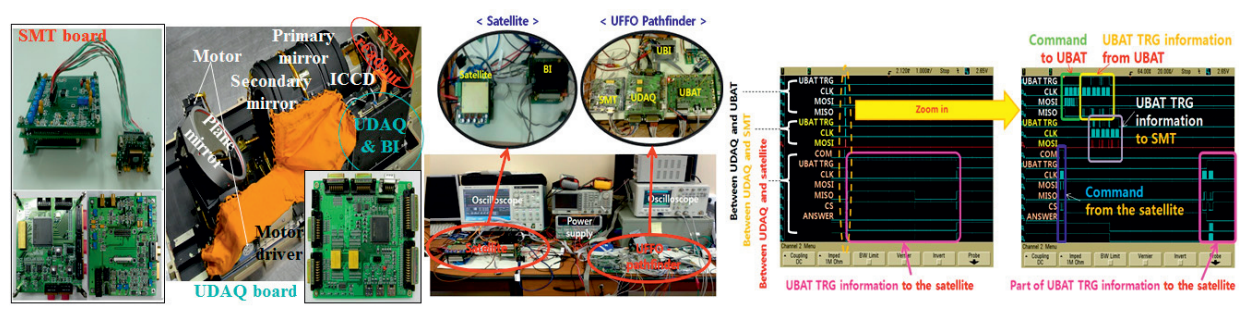

Fig. 3. SMT and UDAQ boards, test setup and test result.

mirror movement commands and data storage are managed by an Actel A3P1000 FPGA.

\subsection{UFFO Data Acquisition (UDAQ)}

Using an Actel A3P1000 FPGA, UDAQ acts as the central control system, which controls and manages the operation and communication of each telescope and is also in charge of the interface with the satellite. It will write the data taken by each telescope in the NOR flash memory and send them to the satellite via the Bus-Interface system (BI), as shown in Figure 3 left. All the operations between UFFO-P and the satellite have been tested (see Fig. 3 right). Interfaces between UDAQ and SMT/UBAT and between UFFO-P and the satellite have been tested too. As shown in Figure 3 right, when UDAQ receives a command for UBAT from the satellite, it first decodes the command and then sends it to UBAT. UBAT also generates and sends the trigger information to UDAQ. Finally UDAQ distributes the information to SMT and the satellite. These UDAQ interfaces including the command and the trigger information show good performance without errors ( $\mathrm{Na}$ et al. 2011).

\section{Conclusion}

UFFO-P is planned to be launched in 2013 and is designed to measure the early phase in particular of short duration gamma ray bursts with a sub-minute response time. The effects of radiation in space on logic device and memory can disrupt performance, resulting in loss of data. Therefore UFFO-P electronics use rad-hard ASICs, military grade Actel FPGA and space grade 3D plus memory for the main components, as shown in Table 1. We are in the final stages of development and tests. So far, we have completed both the electronics and the telescope. The next step is to test perform UBAT- SMT cross calibration.

Table 1. Main components for each system.

\begin{tabular}{cccc}
\hline & SMT & UDAQ & UBAT \\
\hline FPGA (Military) & Actel A3P1000 & Actel A3P1000 & Actel A3PE3000L \\
\hline
\end{tabular}


This research was supported by Basic Science Research Program through the National Research Foundation of Korea (NRF) funded by the Ministry of Education, Science and Technology (2010-0025056), Creative Research Initiatives (RCMST) of MEST/KRF, and supported by World Class University (WCU) program through the National Research Foundation of Korea funded by the Ministry of Education, Science and Technology (R32-2009-000-10130-0) in Korea.

\section{References}

Ahmad, S., Barillon, P., Blin-Bondil, S., et al., 2010, JINST, 5, C12012

Jeong, S., Nam, J.W., Ahn, K.-B., et al., 2013, Opt. Express, 21, 2263

Jung, A., Ahmad, S., Ahn, K.-B., et al., 2011 [arXiv:1106.3802]

Kim, J.E., Ahmad, S., Barillon, P., et al., 2012, ISBN: 9780819491442

Kim, J.E., Lim, H., Jung, A., et al., 2011 [arXiv:1106.3803]

Na, G.W., Ahn, K.-B., Choi, H.S., et al., 2011 [arXiv:1106.3804]

Park, I.H., Brandt, S., Budtz-Jørgensen, C., et al., 2013, New J. Phys., 15, 023031 
